\title{
Danke: Einfach Starten. - Mehr als 2 Millionen mal.
}

\section{Corporate-Service-Design im Packaging für Internet-/TV-Produkte von Kabel Deutschland}

\author{
Dipl.-Des. Oliver Gerstheimer \\ chilli mind $\mathrm{GmbH}$ \\ Königstor 23 \\ 34117 Kassel \\ gerstheimer@chilli-mind.com
}

\section{Dipl.Kffr. Sarah Schuster}

Kabel Deutschland Vertrieb und Service $\mathrm{GmbH}$

Betastraße 6-8

85774 Unterföhring

sarah.schuster@kabeldeutschland.de

\author{
Dipl.-Des. Steffen Wüst \\ chilli mind $\mathrm{GmbH}$ \\ Königstor 23 \\ 34117 Kassel \\ wuest@chilli-mind.com
}

\section{Abstract}

Danke: Einfach Starten. Aufbau in 5 Schritten ... Ein Paket hält, was es verspricht. Dialogisierung, Formatvereinheitlichung und Design-Harmonisierung sind die drei „Helden“ des Service-DesignProjekts für bis zu 2 Mio. versendete Einheiten pro Jahr. Der durchgängig und aktiv geführte Dialog mit dem Kunden macht NICHT Halt an der Umverpackung, sondern setzt sich konsequent und eineindeutig bei Aufbau, Installation und Konfiguration fort - egal, ob analoge oder digitale Medien und Formate betroffen sind.

So wird eine durchgängige „User Experience“ und ein konsistentes „Corporate Service-Design“ über sämtliche Berührungspunkte des Benutzers beim Aufbau von Internet- und TV-Produkten von Kabel Deutschland erreicht: vom Auspackerlebnis über die beigelegten Installations- und Informationsmaterialien, von der verbesserten Lesbarkeit, Lesezeit und Informationskaskade bis zur Optimierung der analogen Anleitungsdokumente und der digitalen Installation Wizards.

\section{Keywords}

Corporate Packaging, Service-Design, User Experience, Benutzerführung, Dialogisierung, Harmonisierung, Vereinheitlichung, Prozessoptimierung, Multi-Stakeholder-Management 


\section{Prolog: Was ist eigentlich Service-Design?}

Die Formel dafür lautet:

\section{Service-Design $=$ analoges und digitales Planen und Entwerfen des Gesamtkontextes + messbare Erlebnis- und Prozessoptimierung bei Endkunden und Auftraggeber}

Während die Ausgaben für Fort- und Weiterbildung im produzierenden Gewerbe bei ca. 2.000 Euro pro Jahr liegen, wird im Dienstleistungssektor gerade mal ein Betrag von durchschnittlich ca. 65 Euro investiert. ${ }^{33}$ Dies zeigt einmal mehr die Notwendigkeit, das Augenmerk auf diesen Bereich zu richten, um auch „Services“ ähnlich erfolgreich weiter zu entwickeln wie das klassische Produkt-Design.

Service-Design unterscheidet sich kaum vom bekannten Produkt-Design. Lediglich das Objekt der Betrachtung ist ein anderes: Befasst sich Produkt-Design zumeist mit industriell gefertigten Gegenständen, so befasst sich Service-Design mit Prozessen, Abläufen oder eben Dienstleistungen - auf Englisch „Services“. Die Techniken, die im Rahmen der Entwicklung angewandt werden sind prinzipiell die Gleichen, allerdings auf die Eigenarten des „virtuellen“ Produkts angepasst. Zunehmend werden auch analoge oder digitale Simulationen im Modellbau genutzt, um Services im wortwörtlichen Sinne erst begreifbar zu machen.

\section{Zielsetzung}

Was auf den ersten Blick aussieht wie die schlichte Optimierung von Produktverpackungen für Unterhaltungselektronik und zugehörige Aufbauanleitungen, ist tatsächlich der gesamtheitliche Entwurf des Systems „Verpackung“: End-2-End-Service-Design vom Versandkarton über den Aufbaukontext bis zur Installation und Nutzung der Kabel Deutschland Endgeräte, also Kabelrouter, HD-Receiver und HD-Video-Recorder.

Ziel und Ergebnis des Projekts waren somit die grundlegende Analyse und Qualitätsevaluation bestehender kundensichtbarer Touchpoints von Kabel Deutschland in den Bereichen TV/Video-on-Demand (TV) und Internet/Telefon (KIP). Zudem sollten die Kundenzufriedenheit verbessert und Installations-/Konfigurationsfehler und -abbrüche verringert werden, um so die Aufwände im After-Sales-Prozess, z. B. durch Hotline- und Service-Calls, zu reduzieren.

End-2-End-Service-Design bedeutet hierbei auch, alle beteiligten Stakeholder im Gesamtprozess - Logistik \& Controlling, Marketing \& Sales sowie Endkunden und Benutzer 
intensiv und iterativ zu beteiligen, um alle Berührungs- und Wahrnehmungspunkte (Customer Touchpoints) auszugestalten.

\section{Umsetzung des Projekts}

Insgesamt war das Projekt in vier aufeinander aufbauende Phasen unterteilt: von der Analyse des Status quo über die Entwicklung und Evaluation des Kommunikationskonzepts und der Einzelmaßnahmen bis zur Umsetzung bzw. dem Transfer zur Produktion der Elemente.

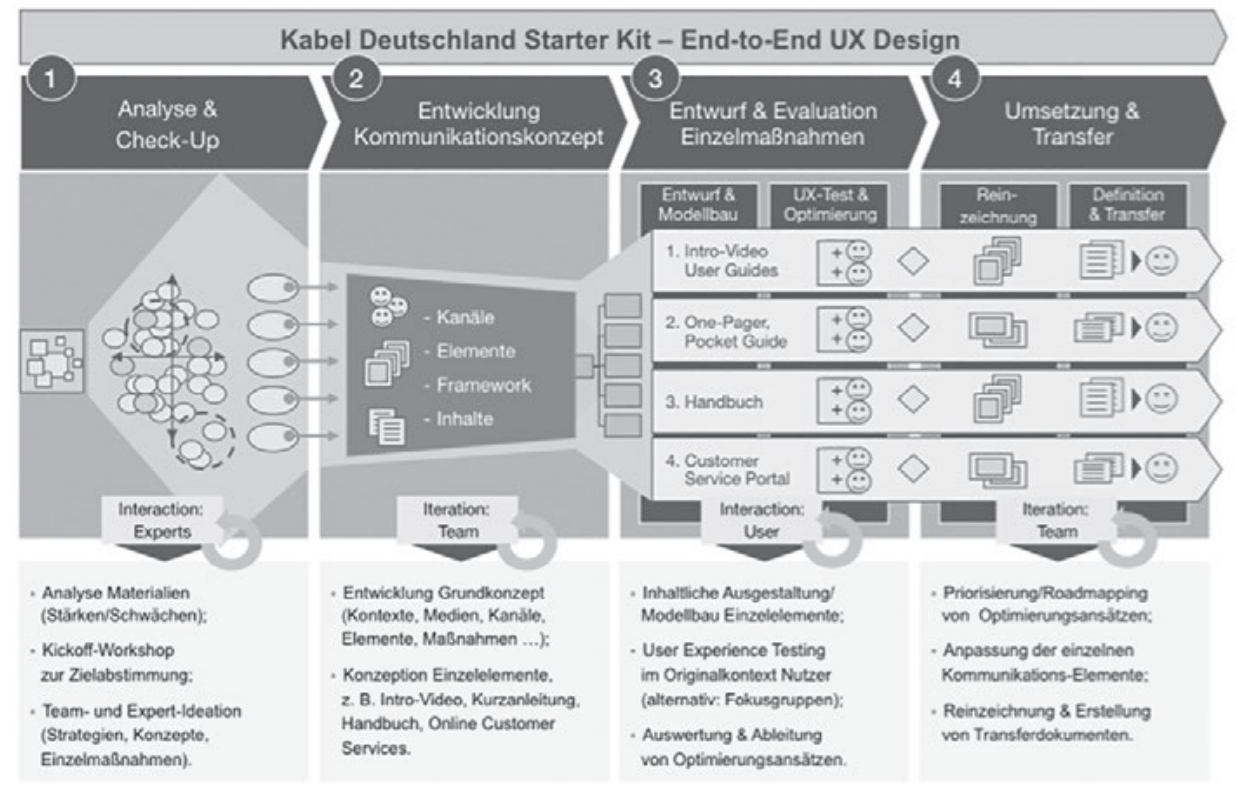

Abbildung 1: Vorgehen im Gesamtprojekt

Ziel der Analysephase 1 war die Erstellung eines priorisierten Anforderungskatalogs aus Nutzersicht auf Grundlage eruierter Bedürfnisstrukturen, resultierend aus kontextuellen Endkunden- und Experten-Interviews, sowie der Analyse aller relevanten Material- und Prozessketten in der Logistik von Kabel Deutschland.

In der Konzeptphase 2 wurden fünf alternative Verpackungskonzepte mit jeweils 12-15 Touchpoint-Elementen entwickelt, die auf 85 identifizierten Optimierungsansätzen aus der Analysephase in 11 Kategorien basierten. Diese wurden sowohl auf Plausibilität aus Benutzersicht, wie auch auf technische und ökonomische Machbarkeit überprüft. Kernstück der Konzeptentwicklung war die Umsetzung einer optimierten Informationsarchitektur und Kaskadierung unter Berücksichtigung der Lese- und Perzeptionszeiten, die aus Benutzersicht den Auspack-, Installations- und Konfigurationsprozess optimal abbildet und unterstützt. Elemente im Gesamtprozess sind (in Klammern jeweils die Perzeptions-/Lesezeiten): 
1. Versandverpackung Außenseite/Innenseite (1-3 Sekunden)

2. Produktverpackung Außenseite/Innenseite (5-10 Sekunden)

3. Informationsmappe mit Willkommensbrief/Zugangsdaten (1-3 Minuten)

4. Kurzanleitung (3-5 Minuten)

5. Gebrauchsanweisung und Software/Installations-Wizard (5-10 Minuten)

Eine weitere Herausforderung für das Konzept stellte die Berücksichtigung der internen Unternehmensprozesse und Anforderungen der Stakeholder dar. In der Evaluationsphase 3 erfolgten die Iterationen der Entwürfe aus diesem Grund in fünf Experten-Fokusgruppen, abteilungsübergreifend mit Produktmanagement, Marketing \& Sales, Controlling \& Logistik sowie Customer-Lifecyle-Management. Im Vordergrund dieser Iterationen standen neben der User Experience, der Gesamtwirkung und Verständlichkeit des Aufbauprozesses aus Benutzersicht auch die Evaluation der Handling-, Logistik- und Produktionskosten. Grundlegende Bewertungskategorien waren:

1. Spontaner Ersteindruck;

2. Kundennutzen und Erlebnis im Aufbauprozess;

3. Logik und Schichtung der Einzelelemente im Versandensemble;

4. Strategie und Brand-Fit, z. B. Durchgängigkeit des 5-Schritt-Aufbaus sowie der Corporate Identity Elemente;

5. Service Promoting Score und Remarkability (Erinnerbarkeit), also positive und weitererzählenswerte Service-Erlebnisse;

6. Umsetzbarkeit (Kostendeckelung, Liefer- und Prozesslogistik im Gesamtprojekt).

Anschließend wurden in der Umsetzungsphase 4 die ausgewählten und spezifizierten Konzepte und Entwürfe durch die jeweiligen Agenturen und Partner von Kabel Deutschland operativ und produktiv umgesetzt.

\section{Ergebnis}

Einige der grundlegenden Herausforderungen des Projekts waren, den gesamtheitlichen Ansatz des Service-Design im Unternehmen nachhaltig zu positionieren, hartnäckig und pragmatisch für ,gute“ Lösungen und Ideen zu kämpfen und Teams sowie Stakeholder dafür zu begeistern. Insbesondere wurden dabei folgende Erkenntnisse gewonnen:

- Durch integrative Formate, wie fachübergreifende Design-Iterationen anhand von alternativen Lösungen, ist auch in einem Projektteam mit 18 Stakeholdern ein effektives und effizientes Vorgehen und argumentatives Entscheiden im Team möglich. 
- Argumentatives Design mit ausgestalteter Varianz objektiviert persönliche und unternehmenspolitische Gestaltungsentscheidungen und schafft so Service-DesignQualität.

- Modellbau ist im Service-Design Trumpf. Zur Vorstellbarkeit von Gesamtkonzept und Einzelelementen werden realitätsnahe und detaillierte Modelle aller User Touchpoints benötigt. Nur so können fachübergreifende Teams profunde Entscheidungen treffen.

- Respekt vor Controlling-, Liefer- und Logistikprozessen! Jeder Cent zählt hier und eine „gesamtheitlich gute“ Gestaltung bekommt eine wirklich herausfordernde Dimension.

Nach Abschluss des Projekts und dem jährlichen Review wurden zudem folgende ,harten Fakten" erhoben und als Projekt-Feedback bzw. -Learning an die Teams zurückgespielt:

- Die Gesamtkosten einer Einheit des Gesamtensembles wurden bei gleichzeitiger Steigerung der Werbewirkung durch Corporate Branding nur um wenige Cent erhöht.

- Die bisher verwendeten neun Paketformate konnten auf drei Formate reduziert werden, was Optimierungen im Handling, der Logistik und im Einkauf zur Folge hatte.

- Im Sinne der Nachhaltigkeit wurde das verwendete Kartonmaterial der Umverpackungen durchgängig auf minimal bedruckte Recycling-Wellpappen vereinheitlicht.

- Die Liefer- und Logistikkette wurde konsequent auf durchgängige Service-Qualität im Sinne des Endprodukts und Service-Design im Sinne des Endkunden ausgerichtet.

- Im Vergleich zu den heterogenen Vorversionen wurde die Auffälligkeit und Merkfähigkeit von Kabel Deutschland Verpackungen nachhaltig erhöht.

- Die optimierte Usability in der Aufbaulogik und der konsequente 5-Schritt-Ansatz haben messbar zur Entlastung der Service-Hotlines beigetragen.

- Und: Das neue Verpackungs-Ensemble wurde im Jahr 2014 ca. 2 Mio. mal versendet

- Das gesamtheitlich entwickelte Projekt wurde Anfang 2015 mit dem iF Design Award für kundenzentriertes Service Design / Packaging in der Rubrik Consumer Products ausgezeichnet.

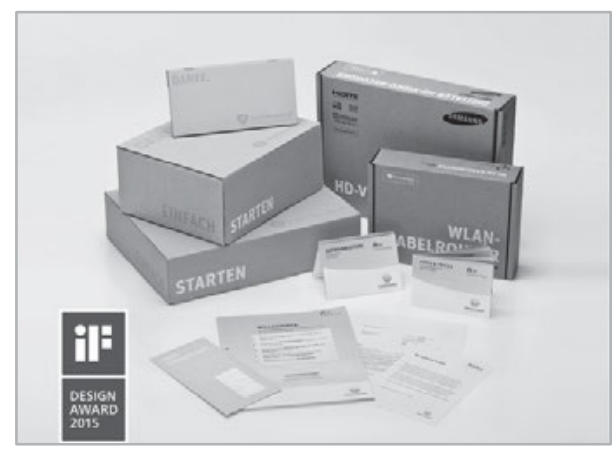

Abbildung 2: Finale Umsetzung des „Ensembles“ 